\title{
Mechanical structural health prognosis with nonlinear mixed frequency ultrasonic signal analysis
}

\author{
Hanxin Chen ", Mingming Liu, Zhenyu Hu, Menglong Li, and Sen Li \\ School of Mechanical and Electrical Engineering, Wuhan Institute of Technology, Wuhan 430074, \\ China
}

Keywords: microdefect, collinear mixing, ultrasonic prognosis, nonlinear coefficient.

\begin{abstract}
In order to detect the early fatigue crack of mechanical components simply, this paper puts forward the ultrasonic testing technology of different side collinear mixing. Firstly, based on the nonlinear ultrasonic theory, the method of calculating the difference frequency and sum frequency nonlinear coefficients of mixing ultrasonic is deduced. Then, the ram-5000 SINAP ultrasonic system is used to detect the aluminum alloy specimens with five different depth fatigue cracks, and the corresponding spectrum diagram is drawn. From the experimental results, we get that the crack depth is positively correlated with the nonlinear coefficients of difference frequency and sum frequency within a certain crack depth. Finally, by analyzing and fitting the experimental data, the prediction models of the difference frequency and sum frequency nonlinear coefficients on the crack depth are established. Through the analysis and combination of the above two prediction models, the prediction model of the mixing relative nonlinear coefficient is established, and the average error of the three prediction models is compared. The results show that the mixing relative nonlinear model has better results. The research work in this paper makes a useful exploration for crack detection and crack depth prediction.
\end{abstract}

\section{Introduction}

Nowadays, metal materials have become the main force of industrial materials. Due to the long-term influence of different loads or high temperature and high pressure environment, the key metal parts of common pressure vessels, transportation pipelines and various engines are very easy to form fatigue cracks on the surface and inside, leading to component fracture failure, and even disaster accidents [1-3]. Therefore, it is very important to detect the early fatigue cracks of key metal parts. Nondestructive testing is the most commonly used and important means to detect material damage. It is of great significance

\footnotetext{
*Corresponding author: pg01074075@163.com
} 
to study the nondestructive testing and evaluation methods of material early damage to ensure its service safety [4].

Ultrasonic nonlinear technology can detect and characterize the early defects of metal parts, and the second harmonic technology is the most commonly used detection method [5]. In the process of the second harmonic test, the test equipment, sensors and couplants will produce higher harmonic, so it is difficult to distinguish the measured ultrasonic nonlinear coefficient effectively. For the conventional ultrasound can not effectively extract the nonlinear coefficient, mixing ultrasonic detection perfectly makes up for this defect. At present, mixing detection method has become a hot direction in the field of ultrasonic nonlinear detection.

Many scholars at home and abroad have done a lot of experimental research on nonlinear mixing theory and experiment. Jones et al. [6] first studied the mechanism of mixing phenomenon in nonlinear medium through theory, solved the nonlinear wave equation in solid medium, and gave five cases of the third wave generated by the interaction of two waves in the medium. Kalyana Sundaram [7-8] published a paper on the nonlinear mixing of two co directional Rayleigh waves. The multi-scale method is used to consider the perturbation expansion including fundamental and second harmonic, sum frequency and difference frequency components. By satisfying the solvability condition, a set of coupled nonlinear differential equations is obtained to perform the numerical simulation.Jiao Jingpin et al. [9-11] used the experimental system of collinear mixing measurement to analyze the bispectrum of the received mixing signal to explore the influence of the excitation frequency on the nonlinear coefficient. In order to study the relationship between ultrasonic nonlinear coefficient and fatigue damage process, Zhang et al. Used harmonic method to detect the fundamental and second harmonic amplitudes of intact aluminum plate, perforated aluminum plate and aluminum plate repaired with composite materials under different fatigue cycles, and then calculated the curve of ultrasonic nonlinear coefficient changing with fatigue cycle.

To sum up, scholars have done a lot of effective research on the mixing nonlinear theory and detection of mechanical component cracks. However, due to the limitations of traditional nondestructive testing methods, this paper studies the nonlinear ultrasonic early defect detection method. The experimental results show that the collinear mixing ultrasonic testing can effectively identify cracks, and get the magnitude of amplitude components corresponding to sum frequency and difference frequency. Then, according to the experimental results and mixing nonlinear coefficient, the relationship between crack depth and nonlinear coefficient is studied.

\section{Nonlinear coefficient analysis of collinear mixing ultrasonic wave.}

The basic principle of mixing method is: input two ultrasonic waves with different frequencies into the tested material at the same time. When the material is in an ideal state, that is, without any defects, the two ultrasonic waves continue to propagate at the original frequency because they meet the principle of linear superposition and do not interfere with each other; when there are small defects in the material, the two ultrasonic fields will interact in the defects, so as to produce a sound wave In this paper, the detection analysis and research are mainly based on the principle of non-linear ultrasonic detection of different side collinear mixing, and the principle is shown in Figure 1. 


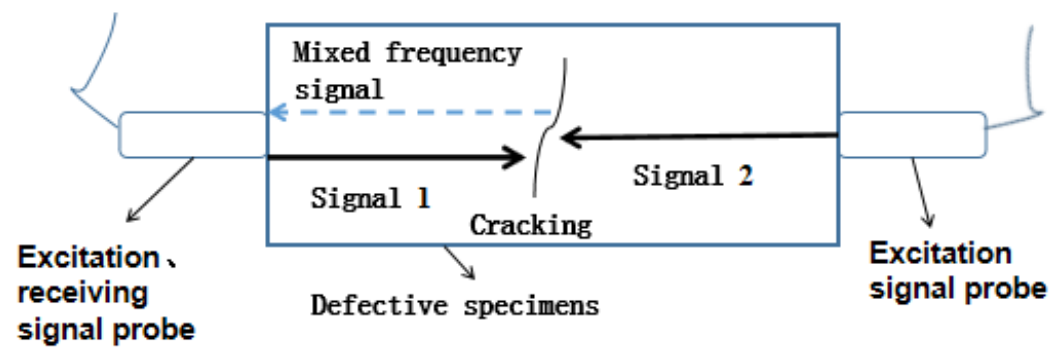

Fig. 1 The principle of hetero-side collinear mixing ultrasonic detection.

When ultrasonic wave propagates in materials with small defects, it will produce nonlinear phenomenon and capture the second harmonic component. When the excited ultrasonic signals are of different frequencies and act together in the material, the nonlinear phenomenon will be different from that under single frequency excitation, and the frequency domain of the received signal will produce beam aliasing. Two kinds of excitation signals with different frequencies are used to excite the material at the same time, which leads to the complex internal sound field coupling phenomenon. The nonlinear wave equation formed in the case of complex sound field coupling can not get the accurate analytical formula and solution. Based on this, this paper solves the nonlinear wave equation without considering the attenuation factor, and ignores the high-order nonlinear coefficients.In metal materials, it is easier to analyze the one-dimensional case. In this paper, one-dimensional longitudinal wave propagating along the direction is taken as an example, and the attenuation is ignored. In isotropic medium, the wave equation is shown in equation (1)

$$
\frac{\partial^{2} u(x, t)}{\partial t^{2}}-c^{2} \frac{\partial^{2} u(x, t)}{\partial x^{2}}=c^{2} \beta \frac{\partial u(x, t)}{\partial x} \frac{\partial^{2} u(x, t)}{\partial x^{2}}
$$

where represents the displacement of the particle, is the propagation distance of the ultrasonic wave, is the wave speed of the ultrasonic wave, and is the ultrasonic nonlinear parameter.Using the theory of perturbation solution of wave equation solution, set for in the form of wave equation solution

$$
u(x, t)=u^{0}(x, t)+\beta u^{1}(x, t)
$$

where represents linear displacement and is the displacement caused by material nonlinearity. If the nonlinear displacement is proportional to the wave propagation distance, then

$$
u^{1}(x, t)=x \omega(\tau)
$$

In equation (6), is an unknown function to be determined, where.If the excitation signal is two ultrasonic waves of different frequencies, the form is

$$
u^{0}(x, t)=A_{1} \cos \left(\omega_{1} \tau\right)+A_{2} \cos \left(\omega_{2} \tau\right)
$$

Among them,and are two excitation signal amplitude,and is the frequency of two excitation signals, and . Plug-in type 2, 3 and 4 will be equation then plug-in type 1 . 


$$
\begin{aligned}
& \omega(\tau)=-\frac{A_{1}^{2} N_{1}^{2}}{8} \cos \left(2 \omega_{1} \tau\right)-\frac{A_{2}^{2} N_{2}^{2}}{8} \cos \left(2 \omega_{2} \tau\right) \\
& +\frac{A_{1} A_{2} N_{1} N_{2}}{4}\left[\cos \left(\omega_{2}-\omega_{1}\right) \tau-\cos \left(\omega_{1}+\omega_{2}\right) \tau\right]
\end{aligned}
$$

And equation (5) can be expressed as

$$
\begin{aligned}
& u(x, t)=A_{1} \cos \left(\omega_{1} \tau\right)+A_{2} \cos \left(\omega_{2} \tau\right) \\
& +\mathrm{x} \beta\left\{\begin{array}{l}
-\frac{A_{1}^{2} N_{1}^{2}}{8} \cos \left(2 \omega_{1} \tau\right)-\frac{A_{2}^{2} N_{2}^{2}}{8} \cos \left(2 \omega_{2} \tau\right) \\
+\frac{A_{1} A_{2} N_{1} N_{2}}{4}\left[\cos \left(\omega_{2}-\omega_{1}\right) \tau-\cos \left(\omega_{1}+\omega_{2}\right) \tau\right]
\end{array}\right\}
\end{aligned}
$$

Among them, and are the wave numbers of the two excitation signals. From Equation (6), we can see that the solution of the wave equation has not only the frequencies and of the excitation signal, but also the second harmonic frequencies and, and the difference frequency signal frequency, and the sum frequency signal frequency . If the amplitude of each frequency signal is known, the calculation formula of the ultrasonic nonlinear coefficient based on mixing technology (referred to as the mixing nonlinear coefficient) is

$$
\beta=\frac{4}{k_{1} k_{2} x} \frac{A_{\left(\omega_{2}-\omega_{1}\right)}}{A_{\left(\omega_{1}\right)} A_{\left(\omega_{1}\right)}}=\frac{4}{k_{1} k_{2} x} \frac{A_{\left(\omega_{1}+\omega_{2}\right)}}{A_{\left(\omega_{1}\right)} A_{\left(\omega_{1}\right)}}
$$

where is the propagation distance of two excitation signals, and difference frequency signals, respectively, and the amplitude and frequency signal. For ease of calculation, the mixing nonlinearity coefficient can be expressed as

$$
\beta=\frac{A_{\left(\omega_{2}-\omega_{1}\right)}}{A_{\left(\omega_{1}\right)} A_{\left(\omega_{1}\right)}}=\frac{A_{\left(\omega_{1}+\omega_{2}\right)}}{A_{\left(\omega_{1}\right)} A_{\left(\omega_{1}\right)}}
$$

Therefore, when the excitation signal point is fixed, the non-linear coefficients of the sum frequency or difference frequency (difference frequency $\beta^{-}$, sum-frequency $\beta^{+}$) measured in the received signal can be studied to realize the defect assessment of the material.

\section{Ultrasound test of blend frequency}

Experimental Setup. The excitation and reception system of the non-linear ultrasonic detection method based on collinear anisotropic mixing is established. The experimental platform device is shown in Fig. 2. The non-linear high-energy ultrasonic system RAM-5000 SINAP acts as a signal generator and the trigger inside the system acts as a reference trigger. Wideband transducer with frequency and narrowband longitudinal wave transducer with frequency are connected to 1 channel and 2 channel of RAM-5000-SNAP respectively. The RDX-6 diplexer is connected to the shear wave transducer so that the shear wave transducer can operate as both a transmitter and a receiver transducer. Stimulate 
and receive sensors. Two ultrasonic waves of different frequencies propagate in the specimen and interact nonlinearly when they meet at the defect to generate a third new wave train, which is simultaneously received by sensor 2 at the left end of the specimen and transmitted to receiver channel 1 of RITEC RAM-5000 system via diplexer.

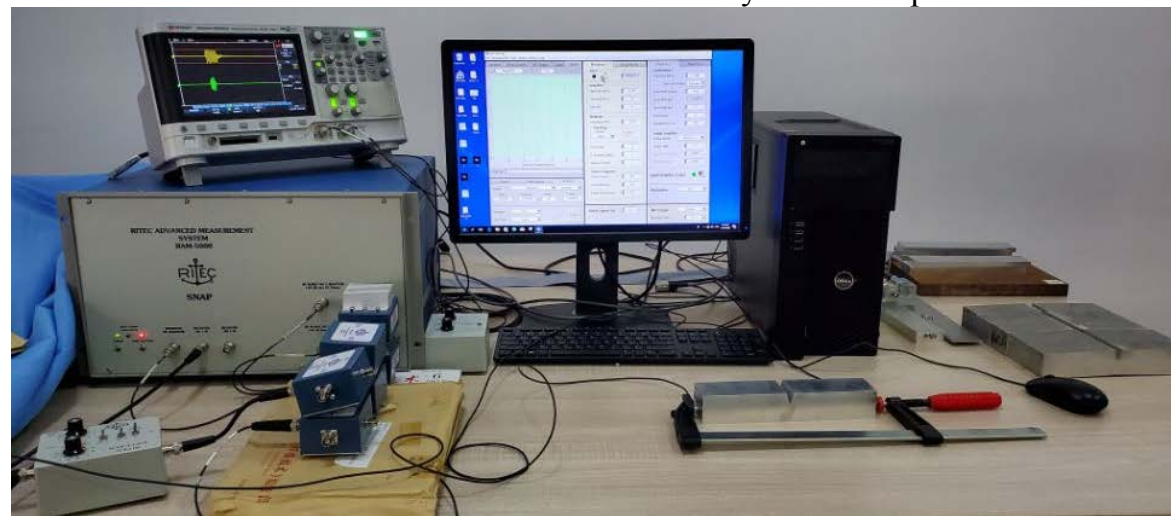

Fig. 2 Experimental platform device.

Selection of Optimum Excitation Signal. Metal fatigue damage detection based on non-linear ultrasonic mixing theory can effectively avoid the non-linear signal interference caused by the system and obtain a more accurate non-linear signal related to damage structure information. However, the target mixing signal extracted and analyzed by the detection method based on this theory is a kind of non-linear effect signal produced in the process of interaction between sound waves and crack. Its energy is very weak, so it is necessary to select the excitation frequency in the experiment. To find the best combination of excitation frequencies for generating obvious mixing signals, the detection and location of damaged structures can be studied.

Combined with the above conditions and the existing ultrasonic frequency in the laboratory, the excitation is made by $2.25 \mathrm{MHz}$ and $5 \mathrm{MHz}$ transducers. After adding a duplexer, the $5 \mathrm{MHz}$ transducer can receive both excitation signals. The frequency response curves of the two transducers are shown in Fig.3.In this experiment, the frequency of two excitation signals is determined as $1.8 \mathrm{MHz}$ and $4.6 \mathrm{MHz}$ respectively. There are two reasons for choosing such a frequency combination:

1) The two fundamental frequency signals have a large frequency difference $(2.8 \mathrm{MHz})$, which distinguishes them well in the frequency domain;

2) Their respective second-order harmonics $(3.6 \mathrm{MHz}$ and $9.2 \mathrm{MHZ})$ differ greatly from each other and do not influence the sum frequency signal $(6.4 \mathrm{MHz})$.
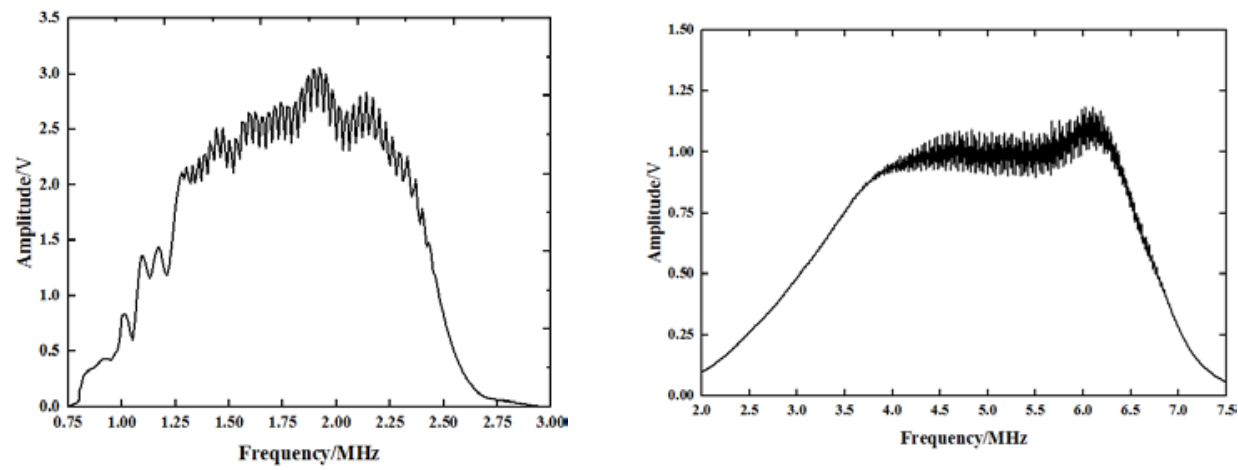

Fig. 3. Frequency response curve. 


\section{Analysis of experimental results}

Experimental results. When the frequency of excitation signal 1 is $1.8 \mathrm{MHz}$ and the frequency of excitation signal 2 is $4.6 \mathrm{MHz}$, collinear mixing tests are carried out on specimens with different depth defects to transmit the received signal to the receiving channel 1 of the ultrasonic non-linear system.Fig. 4 shows the spectrum of the received signal from specimen 5 .

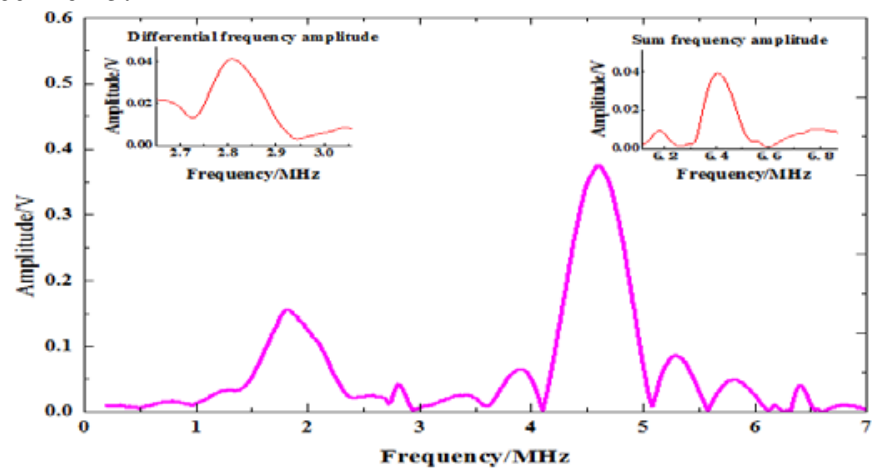

Fig. 4. 5\# specimen received signal spectrum diagram.

From the spectrum Fig. 4 of the received signal in the mixing test, it can be seen that the received signal is complex and contains many frequencies. In addition to the excitation signals at $1.8 \mathrm{MHz}$ and $4.6 \mathrm{MHz}$, there are also signals at $2.8 \mathrm{MHz}$ of different frequency and $6.4 \mathrm{MHz}$ of sum-frequency. Therefore, the frequency selection of the two excitation signals in the co-line mixing test is reasonable. It can be seen from the local amplified frequency domain diagram corresponding to the difference frequency and sum-frequency of the upper end of Fig.4.The amplitude of the differential frequency signal is $A_{\left(\omega_{2}-\omega_{1}\right)}=0.042 \mathrm{~V}$ while that of sum-frequency signal is $A_{\left(\omega_{1}-\omega_{2}\right)}=0.04 \mathrm{~V}$, which is not equal. This is because attenuation exists in the process of ultrasonic transmission and the attenuation is related to the frequency of the signal. Therefore, the attenuation of the amplitude of two mixing frequencies is different, so that the amplitude of differential frequency signal and sum-frequency signal are not identical.

According to the amplitudes of two fundamental wave signals, differential frequency and sum-frequency signals, differential frequency non-linearity coefficient $\beta^{-}$and sum-frequency non-linearity coefficient $\beta^{+}$of five samples under different fatigue life are calculated, then mixing non-linearity coefficient is shown in the table below. To reduce the error of the experiment, each group of samples is repeated three times and their non-linearity coefficient average value is taken.

Table 1. Nonlinear coefficients at different crack depths.

\begin{tabular}{lcc}
\hline Crack depth $/ \mathrm{mm}$ & Differential frequency/ $\beta^{-}$ & Sum frequency/ $\beta^{+}$ \\
\hline 4.1 & 0.2335 & 0.2179 \\
6.918 & 0.2632 & 0.2723 \\
7.624 & 0.3440 & 0.3796 \\
8.505 & 0.3506 & 0.4308 \\
12.215 & 0.7226 & 0.6882 \\
\hline
\end{tabular}



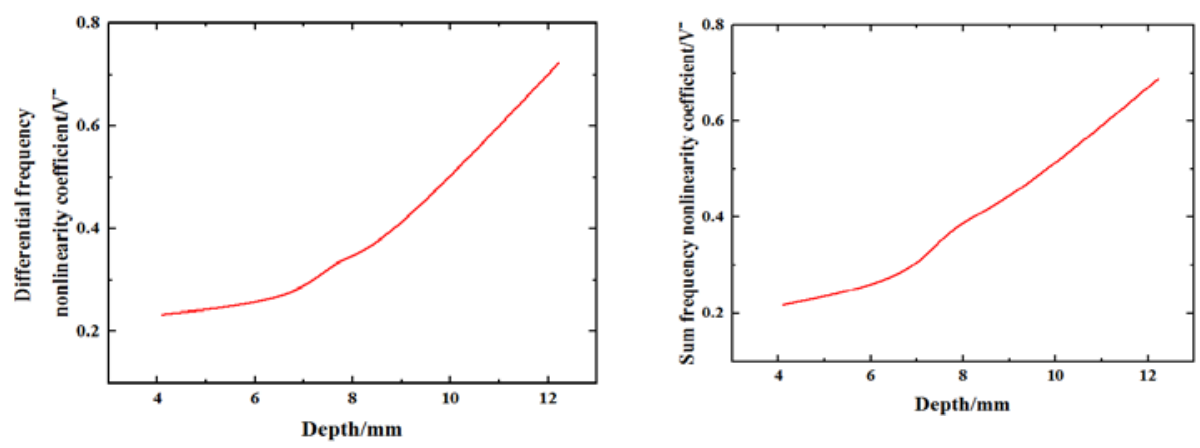

Fig. 5. Nonlinear coefficient versus crack depth.

From Table 1 and Fig.5, it can be seen that with the increase of crack depth, differential frequency non-linearity coefficient $\beta^{-}$and sum frequency non-linearity coefficient $\beta^{+}$ increase monotonously, which reflects that the non-linearity coefficient is positively correlated with crack depth. From the diagram, it can be seen that under certain crack depth, with the increase of crack depth, the increasing trend of non-linear coefficient gradually increases, which is due to the larger contact area of the crack interface, the more obvious the non-linear effect becomes.

Prediction of crack depth in specimens.In the co-linear mixing test, the non-linear coefficient of mixing is closely related to the microstructure of metal under different fatigue conditions, i.e. the crack depth state of the specimen affects the non-linear characteristics of ultrasound. The non-linear coefficient of mixing can effectively assess the crack depth of the material. This correlation provides a basis for the prediction of crack depth of metal specimens.Based on the experimental data in Table 1, the corresponding equation curve between crack depth and nonlinear coefficient $\left(\beta^{-} 、 \beta^{+}\right)$is fitted by Origin software.

$$
\begin{gathered}
H=-46.17 \beta^{-^{2}}+59.28 \beta^{-}-6.53 \\
H=-16.67018 \beta^{+^{2}}+3.104273 \beta^{+}-1.2922
\end{gathered}
$$

The complex correlation coefficient $\mathrm{R}$ is used to characterize the correlation between the fitting equation and actual value. The closer the fitting effect of one equation is, the better the fitting effect is. $R>0.8$ means that there is a strong correlation between the two equations. The complex correlation coefficient $R$ of the non-linear coefficient $\beta^{-} 、 \beta^{+}$is 0.93337 and 0.9126 respectively. Therefore, the two fitting equations have a good fitting effect.

From the fitting curve between mixing nonlinear coefficient and crack depth of specimen, it can be seen that there is an approximate quadratic fitting relationship between mixing nonlinear coefficient and crack depth. Therefore, this fitting curve can be used as a model to predict the crack depth of metal specimens based on collinear mixing technology.

To emphasize the relationship between mixing nonlinear coefficient and crack depth of specimens, a relative characteristic parameter - mixing non-linear relative parameter $\hat{\beta}$ is proposed to predict the crack depth of specimens based on data fusion theory. Since both 
the differential-frequency nonlinearity coefficient and sum-frequency nonlinearity coefficient are proportional to the amplitude of differential/sum-frequency signals received by ultrasound and have the same dimensions, the mixed-frequency nonlinearity comprehensive parameter is defined as $\hat{\beta}=\beta^{-} \times \beta^{+}$.

Based on the experimental data in Table 1, the fitting curve equation between the mixing nonlinear relative parameter $\hat{\beta}$ and the crack depth $H$ of the specimen is:

$$
H=-57.77028 \hat{\beta}^{2}+47.9898 \hat{\beta}+2.62737
$$

The fitting complex correlation coefficient indicates that the fitting curve equation can better reflect the relationship between the non-linear relative parameters of mixing frequency and the crack depth of the specimen, and the relationship is shown as follows by Fig. 6.

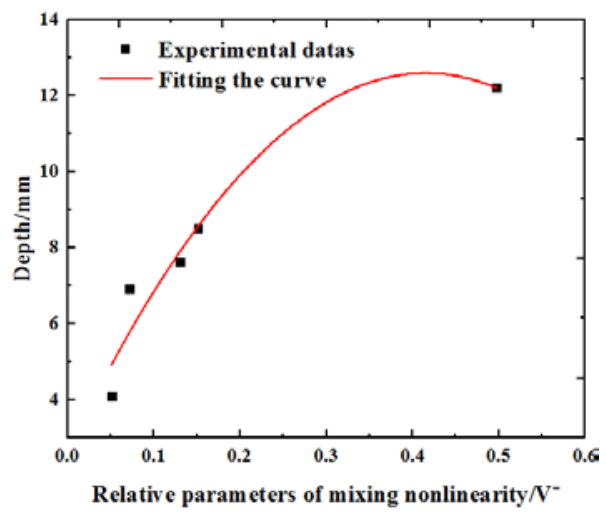

Fig. 6. Relationship between nonlinear relative parameters of mixing and crack depth.

The above fitting curve equations are all based on the mixing ultrasonic test data. Through the experimental data and Origin Software fitting, the difference frequency nonlinear coefficient, sum-frequency nonlinear coefficient and mixing nonlinear relative parameter and under different crack depths are calculated, and the relationship between and crack depth is predicted respectively. The prediction results are shown in Fig.7.

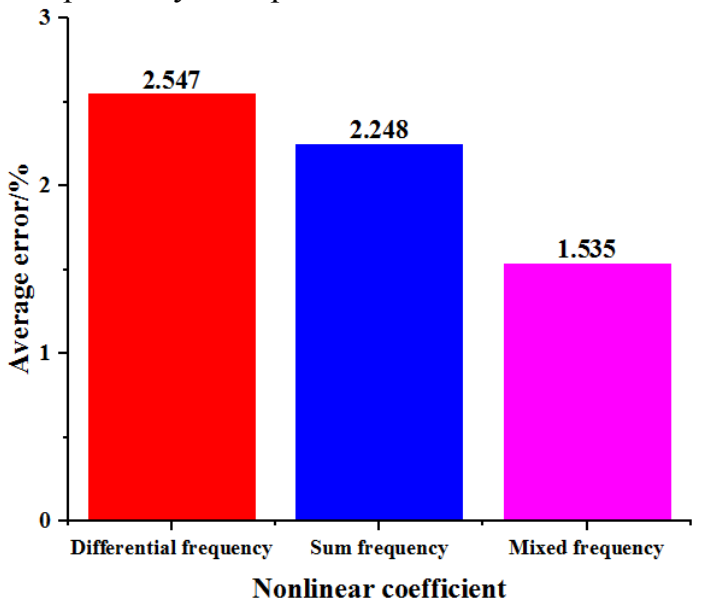

Fig. 7. Prediction of crack depth by the nonlinear coefficient. 
It can be seen from Fig.7 that from the analysis of the average error of crack depth prediction of a difference frequency, sum-frequency nonlinear coefficient and mixing nonlinear relative coefficient, the above fitting curve equation can well characterize the relationship between nonlinear coefficient and crack depth, but the average error of prediction based on mixing nonlinear relative coefficient is the smallest, which indicates that the prediction based on mixing nonlinear relative parameter is feasible The measurement model can fully show the dependence of the crack depth on the nonlinear mixing coefficient.

\section{Conclusion}

In this paper, the different side collinear mixing ultrasonic testing technology is used to study the cracks of mechanical components. The conclusions are as follows

The aluminum alloy specimens with different depth cracks were tested by ram-5000 SINAP. The results show that in a certain range of crack depth, the crack depth is positively correlated with the nonlinear coefficients of different frequency and sum-frequency.

Based on the experimental data, the prediction models of a difference frequency, sum-frequency nonlinear coefficient $\left(\beta^{-} 、 \beta^{+}\right)$and mixing relative nonlinear coefficient $(\hat{\beta})$ to crack depth are obtained, and the prediction model of mixing relative nonlinear coefficient can better predict the crack depth of corresponding specimens.

\section{References}

1. Chen Hanxin, Fan Dongliang, Huang Jinmin, et al. Finite element analysis model on ultrasonic phased array technique for material defect time of flight diffraction detection[J]. Science of advanced material 12(5)(2020), $665-675$.

2. Y.J. Li, Q.C. Chang, et al. Complex data study on mechanical fault diagnosing[J]. Journal of Intelligent \& Fuzzy Systems, 2018, 34(2), 1169-1176.

3. Chen Hanxin, Huang Wenjian, Huang Jinmin, et al. Multi-fault condition monitoring of slurry pump with principle component analysis and sequential hypothesis test[J].International Journal of Pattern Recognition and Artificial Intelligence (2019), https://doi.org/10.1142/S0218001420590193.

4. Hanxin Chen, Dong Liang Fan,et al. Particle swarm optimization algorithm with mutation operator for particle filter noise reduction in mechanical fault diagnosis[J]. International journal of pattern recognition and artificial intelligence (2020), https://doi.org/10.1142/S0218001420580124.

5. Hanxin Chen, et al. Model-based method with nonlinear ultrasonic system identification for mechanical structural health assessment[J], Transactions on emerging telecommunications technologies (2020), e3955, http://doi.org/10.1002/ett.2955.

6. Jones GL, Kobett D R. Interaction of Elastic Waves in an Isotropic Solid[J]. Journal of the Acoustical Society of America, 1963, 35(1):5-10

7. Kalayanasundaram N. Nonlinear mode coupling of surface acoustic waves on an isotropic solid[J]. International Joumal of Engineering Science, 1981, 19(3):435-441

8. Kalyanasundaram N. Nonlinear mixing of surface acoustie waves propagating in opposite directions[J]. Journal of the Acoustical Society of America, 1998, 73(6):1956-1965. 
9. Walker S V, KimJ Y, Qu J, et al. Fatigue damage evaluation in A36 steel using nonlinear Rayleigh surface waves[J]. NDT \& E International, 2012.48(2):10-15.

10. Lim H J, Sohn H. Fatigue crack detection using structural nonlinearity reflected on linear ultrasonic features[J]. Jounal of Applied Physics, 2015, 118(24):244902.

11. Chen H, Zhang G, Fan D, et al. Nonlinear Lamb Wave Analysis for Microdefect Identification in Mechanical Structural Health Assessment[J]. Measurement, 2020, 164:108026.

12. Jiao J, Sun J, Li N, et al. Micro-crack detection using a collinear wave mixing technique[J].NDT\& E International, 2014, 62(2):122-129.

13. Chen Z. Tang G. Zhao Y, et al. Mixing of collincar plane wave pulses in elastic solids with quadratic nonlinearity[J]. Journal of the Acoustical Society of America, 2014,136(5):2389-2404.

14. Zhang Fayou, Li Cheng, Tie Ying, et al. Experimental study on fatigue damage detection based on nonlinear ultrasonic [J] FRP composites 2019 (04): 12-16 + 30

15. J.-Y. Kim, J. Qu, L. J. Jacobs, J. W. Littles, M. F. Savage. Acoustic Nonlinearity Parameter Due to Microplastic ity. Journal of Nondestructive Evaluation, 2006, 25(1):29-37. 